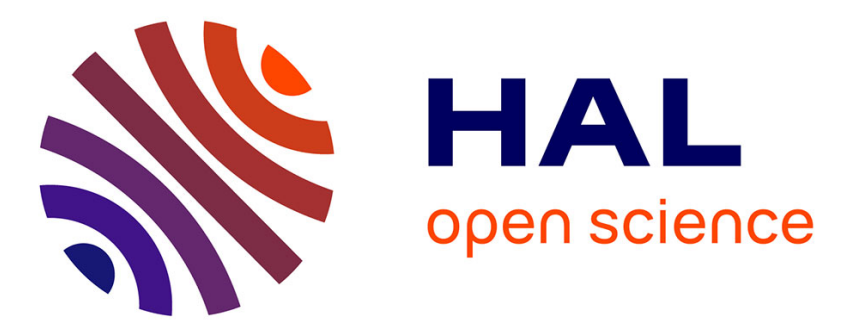

\title{
A framework for the correction of slow physiological drifts during MR-guided HIFU therapies: Proof of concept
}

Cornel Zachiu, Baudouin Denis de Senneville, Chrit T. W. Moonen, Mario Ries

\section{To cite this version:}

Cornel Zachiu, Baudouin Denis de Senneville, Chrit T. W. Moonen, Mario Ries. A framework for the correction of slow physiological drifts during MR-guided HIFU therapies: Proof of concept. Medical Physics, 2015, 42 (7), pp.4137-4148. hal-01159816

\section{HAL Id: hal-01159816 https://hal.science/hal-01159816}

Submitted on 3 Jun 2015

HAL is a multi-disciplinary open access archive for the deposit and dissemination of scientific research documents, whether they are published or not. The documents may come from teaching and research institutions in France or abroad, or from public or private research centers.
L'archive ouverte pluridisciplinaire HAL, est destinée au dépôt et à la diffusion de documents scientifiques de niveau recherche, publiés ou non, émanant des établissements d'enseignement et de recherche français ou étrangers, des laboratoires publics ou privés. 
A framework for the correction of slow physiological drifts during MR-guided HIFU therapies: Proof of concept

Cornel Zachiu

Imaging Division, UMC Utrecht, Heidelberglaan 100, 3584 CX, Utrecht,

Netherlands

Baudouin Denis de Senneville

Imaging Division, UMC Utrecht, Heidelberglaan 100, 3584 CX, Utrecht,

Netherlands and Mathematical Institute of Bordeaux, University of Bordeaux,

33405 Talence Cedex, France

10

Chrit Moonen

Imaging Division, UMC Utrecht, Heidelberglaan 100, 3584 CX, Utrecht, Netherlands

Mario Ries

Imaging Division, UMC Utrecht, Heidelberglaan 100, 3584 CX, Utrecht, Netherlands

(Dated: 22 April 2015) 
Purpose: While respiratory motion compensation for MR-guided HIFU interventions has been extensively studied, the influence of slow physiological motion due to, for example, peristaltic activity, has so far been largely neglected. During lengthy interventions, the magnitude of the latter can exceed acceptable therapeutic margins. The goal of the present study is to exploit the episodic work-flow of these therapies to implement a motion correction strategy for slowly varying drifts of the target area and organs at risk over the entire duration of the intervention.

Methods: The therapeutic work-flow of an MR-guided HIFU intervention is in practice often episodic: Bursts of energy delivery are interleaved with periods of inactivity, allowing the effects of the beam on healthy tissues to recede and/or during which the plan of the intervention is re-optimized. These periods usually last for at least several minutes. It is at this time scale that organ drifts due to slow physiological motion become significant. In order to capture these drifts we propose the integration of 3D MR scans in the therapy work-flow during the inactivity intervals. Displacements were estimated using an optical flow algorithm applied on the 3D acquired images. A preliminary study was conducted on 10 healthy volunteers. For each volunteer 3D MR images of the abdomen were acquired at regular intervals of $10 \mathrm{~min}$ over a total duration of $80 \mathrm{~min}$. Motion analysis was restricted to the liver and kidneys. For validating the compatibility of the proposed motion correction strategy with the work-flow of an MR-guided HIFU therapy, an in-vivo experiment on a porcine liver was conducted. A volumetric HIFU ablation was completed over a time span of $2 \mathrm{~h}$. A 3D image was acquired before the first sonication, as well as after each sonication.

Results: Following the volunteer study, drifts larger than $8 \mathrm{~mm}$ for the liver and $5 \mathrm{~mm}$ for the kidneys prove that slow physiological motion can exceed acceptable therapeutic margins. In the animal experiment motion tracking revealed an initial shift of up to $4 \mathrm{~mm}$ during the first $10 \mathrm{~min}$ and a subsequent continuous shift of $\sim 2 \mathrm{~mm} / \mathrm{h}$ until the end of the intervention. This leads to a continuously increasing mismatch of the initial shot planning, the thermal dose measurements and the true underlying anatomy. The estimated displacements allowed correcting the planned sonication cell cluster positions to the true target position, as well as the thermal dose estimates during the entire intervention and to correct the NPV-measurement. 
A spatial coherence of all three is particularly important to assure a confluent ablation volume and to prevent remaining islets of viable malignant tissue.

Conclusions: This study proposes a motion correction strategy for displacements resulting from slowly varying physiological motion that might occur during an MR-guided HIFU intervention. We have shown that such drifts can lead to a misalignment between interventional planning, energy delivery and therapeutic validation. The presented volunteer study and in-vivo experiment demonstrates both the relevance of the problem for HIFU therapies and the compatibility of the proposed motion compensation framework with the work-flow of a HIFU intervention under clinical conditions.

Keywords: motion correction, MR-guidance, non-invasiveness, HIFU, slow physiological drifts. 


\section{INTRODUCTION}

Magnetic resonance guided high intensity focused ultrasound (MRg-HIFU) is an attractive solution for treating tumors inside the human body in a non-invasive manner ${ }^{1,2}$. Such interventions have the potential to target and ablate non-invasively primary and metastatic cancer deep in the human body in a single session ${ }^{3}$. Due to the associated reduced burden for the patient, treatment related patient morbidity and complication rate, HIFU represents a potential alternative to patient groups which are currently not eligible for invasive or minimally invasive therapy ${ }^{4,5}$. MRg-HIFU has been successfully applied for a number conditions such as symptomatic uterine fibroids, prostate cancer, liver tumors, thyroid nodules, palliative treatment for bone metastases, breast fibroadenoma and functional neurological disorders, including essential tremor, neuropathic pain and tremor-dominant Parkinson's disease $^{5}$. However, MRg-HIFU in clinical applications becomes challenging when the tumor is located in the abdomen or the lower thorax due to continuous physiological motion ${ }^{6-10}$. Uncertainties regarding the exact tumor position might lead to undesired thermal damage to healthy tissue or the delivery of a thermal dose to the tumor that is below the lethal threshold ${ }^{6}$. In addition to shifts caused by respiration, tumors in the abdomen and the lower thorax will also suffer displacements due to digestive and metabolic processes, abdominal and thoracic muscle relaxation and ultimately with the spontaneous motions that the patient might exhibit. Important is to realize that these types of motion do, in general, not occur on the same time-scale:

- Respiratory induced motion of free-breathing patients has a frequency of $0.2-0.4 \mathrm{~Hz}$ and displays a rapid in and exhalation of a duration of 1-1.5 s followed by a rest of the diaphragm of 2-3 times of this duration. The majority of the existing scientific work on motion correction schemes for external beam-interventions focuses on the correction of this type of motion ${ }^{11,12}$.

- Although the time-scale of digestive, metabolic or muscle relaxation motion events depends on the event source (e.g. development of digestive gases or the passage of digestive products in the gastrointestinal tract, bladder filling with urine, changes in patient respiration), the resulting shifts in the position of abdominal organs has in most studies been identified to become significant on a time-scale of several minutes ${ }^{13-15}$. 
For this reason this type of motion will be referred to in this paper as slow physiological motion or drifts. Digestive activity can clinically be moderated by several measures: Peristaltic bowel motion and the development of digestive gases can be of the image volumes, which are both compatible with the therapeutic work-flow of an 
MRg-HIFU therapy. Subsequently, we assess a lower boundary of the accuracy of the proposed co-registration methods for both organs based on a comparison with manually tracked anatomical landmarks at selected areas. Finally, we demonstrate the clinical benefit of the proposed approach by correcting for the slowly varying organ drifts during an in-vivo high intensity focused ultrasound ablation on a porcine liver.

A similar study was recently conducted by Schmitt et al. [REF] in which temperature and thermal dose measurements were corrected with respect to susceptibility artifacts arising from bowel motion during MRg-HIFU interventions in the prostate. While we propose a correction strategy based on displacements provided by a motion estimation algorithm, their study focuses on developing and implementing a filter that excludes temperature information in regions that manifest a higher temperature fluctuation than the heated area. The approach was validated in five out of six clinical cases showing good potential for further use not only in the prostate but also for targets situated higher in the abdomen.

\section{MATERIALS AND METHODS}

\section{II.A. General strategy}

\section{II.A.1. Proposed MR imaging framework}

Figure 1 details the HIFU ablation protocol employed in our study. Our framework consists of performing a series of $n$ ablative sessions, each one having an approximate duration of 5-10 minutes (which is divided between the sonication and a cool-down interval). Each sonication is usually preceded by a planning/re-planning phase, which consists in establishing/refining the position and size of the area that needs to be ablated by that respective sonication. This step is typically performed on a separate high-resolution T1/T2 weighted image acquired at the beginning of the therapy. Note that the MR acquisition sequence for the planning image might differ from the one of the subsequent 3D T1-weighted abdominal scans (see Figure 1). During the actual sonications, MR thermometry is performed in order to monitor the temperature of the sonicated tissue and detect when the lethal thermal dose has been delivered. In this study we were interested in tracking the liver and kidneys over the entire duration of the intervention. At this timescale the organs may exhibit significant displacements in all three directions. Thus, organ tracking should be performed in 3D. With 
current technology this becomes, however, a challenging task during sonications due to the real-time feedback loop required for temperature monitoring ${ }^{6}$. Such a time constraint does currently not allow the acquisition of 3D images with reasonable quality coupled with a 3D image based tracking procedure. For this reason imaging and tracking during sonications has to be performed in 2D. During this time interval the focus is mainly on compensating for displacements caused by respiratory motion. In order to extend liver and kidney tracking to the duration of the entire therapy, we propose interlacing 3D T1-weighted abdominal scans in between sonications. These scans are evenly distributed over the time span of the therapy with a time gap $T_{a}$ in between them. Registration of the acquired $3 \mathrm{D}$ volumes to a reference position allows compensation of the 3D displacements exhibited during sonication by the targeted organs due to slow physiological motion. The acquisition and registration of the $3 \mathrm{D}$ volumes in between sonications is timewise feasible. This is due to the fact that each sonication is followed by a time interval within which the tissue situated in the near and far field of the HIFU transducer is allowed to cool down. The displacements estimated during the $3 \mathrm{D}$ registration process are then used to locate the ablated tissue on a $3 \mathrm{D}$ reference volume.

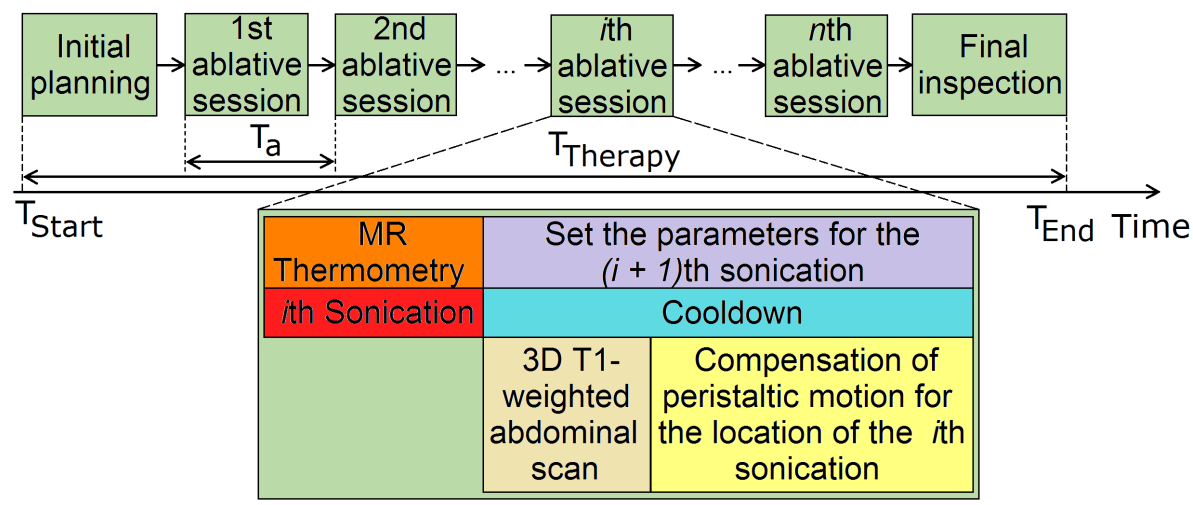

FIG. 1 HIFU ablation protocol employed in our study. The 3D T1-weighted abdominal scans performed at regular time intervals allow the estimation of the 3D displacements exhibited by liver and kidneys due to slow physiological motion. 


\section{II.B. Estimation of 3D organ deformation}

The 3D organ deformations were estimated after each sonication using an optical flow algorithm applied on the T1-weighted volumetric scans. The deformation estimation is based on the optical flow algorithm proposed by Horn\&Schunck ${ }^{28}$. In its original form the algorithm is unable to estimate displacements that are larger than the size of one voxel. In order to overcome this limitation, a multi-resolution approach with an iterative refinement of the optical flow has been employed ${ }^{29}$. A multi-threaded $\mathrm{C}++$ implementation of this algorithm has been provided by Team MR-HIFU, UMC Utrecht, The Netherlands. The algorithm requires as input two images: a reference image and an image to register. For the volunteer study and also for the animal experiment, the first image in their corresponding series of 3D T1-weighted abdominal scans was established as reference. Additionally, the optical flow algorithm requires fixating a parameter $\alpha$ which reflects the elasticity of the tracked organs. All 3D images were registered using $\alpha=0.05$, value which was established empirically.

Given that the optical flow algorithm is gray-level intensity based ${ }^{28}$, it is important to evaluate its robustness to the noise that might superimpose on the images during the acquisition process. For this purpose, registration has been performed between two un-altered 3D T1-weighted abdominal scans and the resulting motion vector field was established as gold standard. Afterwards, noise was added to the image to register, gradually reducing its signal-to-noise ratio (SNR). Registration was then performed for each noise level and a mean square error(MSE) between the current estimated motion vector field and the gold standard was computed. Such an approach will aid in detecting the lowest value of the SNR for which the estimated motion is still reliable.

\section{II.C. Experimental setup}

All MR imaging was performed on a 1.5T Philips Achieva scanner (Philips Healthcare, Best, The Netherlands).

\section{II.C.1. Healthy volunteer study}


The slowly varying drifts exhibited by the human liver and kidneys have been assessed for ten healthy volunteers. All volunteers went inside the MR scanner in a head first prone position and were instructed not to move over the duration of the image acquisition process. A series of nine 3D T1-weighted scans were performed on the abdomen of each volunteer. Timewise the scans were evenly distributed over a duration of approximately $T_{\text {Therapy }}=80$ minutes with an average time gap of $T_{a}=10$ minutes in between the scans. The MR acquisition sequence was a fat suppressed spoiled gradient recalled echo employing the following parameters: $\mathrm{TE}=2 \mathrm{~ms}, \mathrm{TR}=4.3 \mathrm{~ms}$, image matrix $192 \times 192 \times 75,10^{\circ}$ flip angle, bandwidth in phase-encoding direction $373.4 \mathrm{~Hz}$, an isotropic voxel size of $2 \times 2 \times$ $2 \mathrm{~mm}^{3}$, resulting an acquisition time of $\sim 90 \mathrm{~s}$. Fat suppression was achieved through the spectral presaturation with inversion recovery technique. For the remainder of this paper the images acquired using the MR sequence with the aforementioned parameters will be referred to as 3D anatomical images. An exception to this nomenclature will be made for the $3 \mathrm{D}$ anatomical images that play the role of reference in the registration process. These images will be refered to as 3D anatomical anchors.

\section{II.C.2. Animal experiment}

HIFU ablation has been performed in-vivo on a porcine liver using a Sonalleve (Philips Healthcare, Helsinki, Finland) ablation system. The pig was anesthetized by an initial intramuscular injection containing ketamine $(13 \mathrm{mg} / \mathrm{kg})$, midazolam $(0.7 \mathrm{mg} / \mathrm{kg})$, atropine $(0.02 \mathrm{mg} / \mathrm{kg})$ and meloxicam $(0.4 \mathrm{mg} / \mathrm{kg})$. Subsequently, anesthesia was maintained by intravenous administration of sufentanil $(11.3 \mu \mathrm{g} / \mathrm{kg} / \mathrm{h})$, midazolam $(1 \mathrm{mg} / \mathrm{kg} / \mathrm{h})$ and nimbex $(0.09 \mathrm{mg} / \mathrm{kg} / \mathrm{h})$. A total number of $n=10$ sonications were carried out over an approximate duration of $T_{\text {Therapy }}=2$ hours. After each sonication a $3 \mathrm{D}$ anatomical image has been acquired. An additional 3D anatomical image was acquired before the first sonication, which will play the role of reference during the registration process. Closely after the acquisition of the last 3D anatomical image, a gadolinium based contrast agent has been administered to the animal. Subsequently a contrast enhanced T1-weighted image was acquired. In this manner, a visual feedback of the non-perfused tissue resulting from the 10 sonications is obtained. After the completion of the experiment, the animal was euthanized by intravenous injection of pentobarbital. 
The animal experiment was conducted in agreement with the European law on animal experimentation and in compliance with the institution's rules for animal care and use.

\section{II.D. Analysis and validation of the $3 \mathrm{D}$ estimated motion}

\section{II.D.1. Motion analysis and validation on healthy volunteers}

In this study we were interested exclusively in the motion exhibited by the liver and kidneys. In order to restrict motion analysis to the mentioned organs of interest, a semiautomatic segmentation procedure was performed prior to the analysis. An initial segmentation was obtained by the means of active contours which was subsequently refined through a manual correction. The software tool used for segmentation was ITK-Snap v 2.4.0.

The validity of the estimated motion was tested by manually tracking blood vessel bifurcations in the liver and kidneys. For each of the volunteers three vessel bifurcations were tracked: one in the upper part of the liver (lobes 7 and 8), one in the lower part of the liver (lobes 5 and 6 ) and one in the kidneys. The manually tracked landmarks were then compared to the displacements estimated by the optical flow algorithm.

The manually measured displacements were also used to quantify the positional errors caused by the slowly varying drifts in the absence and respectively in the presence of a motion compensation strategy. This was achieved by comparing the displacements obtained through manual tracking (which correspond to the positional errors we would have in the absence of a motion compensation scheme) with the difference between these displacements and the displacements estimated by the optical flow algorithm (which corresponds to the residual positional errors when a motion compensation scheme is integrated in the therapy workflow). Note that for computing the positional errors, the magnitude of the 3D displacement vectors was used instead of separately evaluating the errors on each of the three directions of motion.

\section{II.D.2. Motion analysis and validation for a real HIFU therapy on an animal}

As a result of slow physiological motion, the porcine liver might suffer displacements during the HIFU ablation session. By using the acquired 3D anatomical images, the position 
of the sonicated areas was retrospectively tracked over the time span of the session. This allowed identifying on the planning image, acquired at the beginning of the HIFU intervention, the actual anatomy that was ablated, which, due to the organ drifts, might differ from the one originally planned. The estimated motion was also used to bring the temperature maps acquired during the 10 sonications to a common reference space determined by the planning image. In this reference space, the delivered thermal dose ${ }^{30}$ was re-calculated. This resulted in a correction such that the newly calculated thermal dose will match the anatomy captured by the planning image. Additionally, the position of the non-perfused volume (NPV) resulting from the intervention was also brought in the reference space of the planning image.

Similar to the healthy volunteer study, motion analysis for the animal experiment has been restricted to the sonicated organ. For this purpose a segmentation procedure as the one described in section II.D.1 has been conducted.

\section{RESULTS}

\section{III.A. Measured displacement for liver and kidneys caused by slow} physiological drifts motion in one of the volunteers abdomen. The displayed figures correspond to a coronal (figure 2(a)) and respectively a sagittal (figure 2(b)) slice extracted from a set of images obtained by subtracting from the 3D anatomical anchor subsequent 3D anatomical images. The leftmost images in the two figures depict the anatomy present in the two slices as captured by the 3D anatomical anchor. In this particular data set, considering just the liver and kidneys, the superior part of the liver experiences an obvious displacement in the craniocaudal direction. The peak of this displacement takes place at the acquisition time of the last 3D anatomical image. Given the image's voxel size of $2 \times 2 \times 2 \mathrm{~mm}^{3}$, this displacement is measured to be approximately $13.5 \mathrm{~mm}$. In comparison, from a visual point of view, the displacements in the left-right and anterior-posterior directions were deemed to be negligible. 


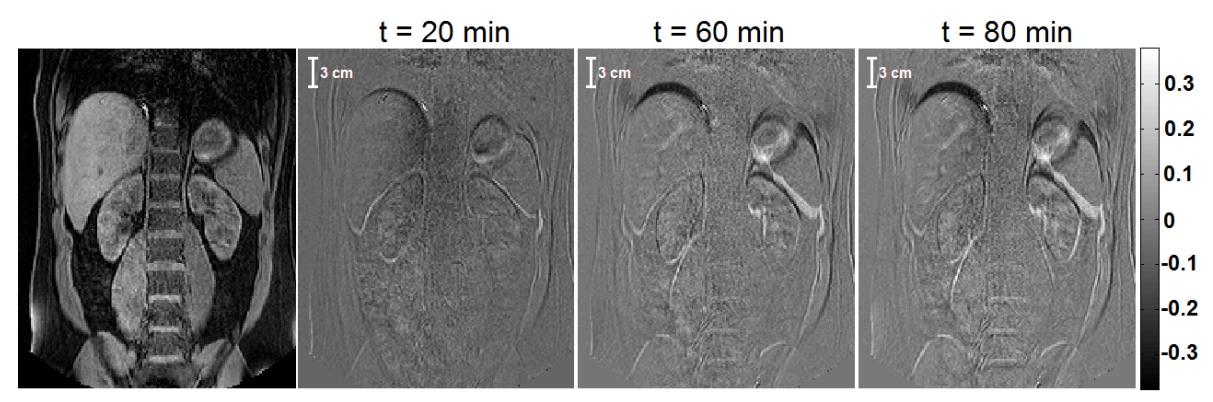

(a)Coronal slice
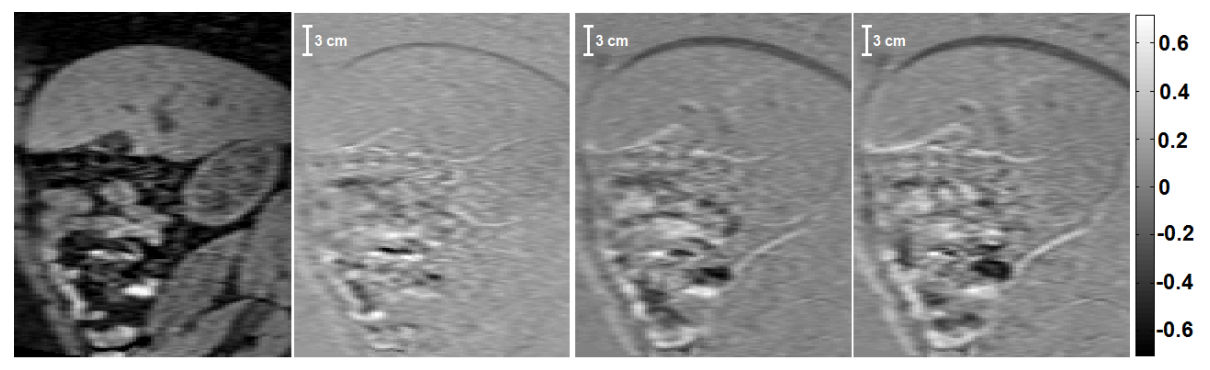

(b)Sagittal slice

FIG. 2 Set of images that result from the subtraction between the 3D anatomical anchor and the $3 \mathrm{D}$ anatomical image acquired $t$ minutes after the acquisition of the anatomical anchor in one of the volunteers. The figures correspond to a (a) coronal (b) sagittal slice from the resulting 3D difference images. The leftmost slices give a visual depiction of the anatomy present in the difference images.

\section{III.B. Robustness to noise of the optical flow algorithm}

Figure 3 displays the relationship between the SNR of the image to register and the MSE of the corresponding estimated vector field with respect to the gold standard. It can be observed that for SNR values less than 5 errors start increasing exponentially. Thus, for images with an SNR value bellow this threshold, the estimated motion vectors are to be considered unreliable.

\section{III.C. Quantitative analysis of the motion amplitude}

For each volunteer the output of the registration process was a set of eight motion vector fields, each corresponding to the displacements estimated at the acquisition time of their associated 3D anatomical image. The motion fields from the individual volunteers were 


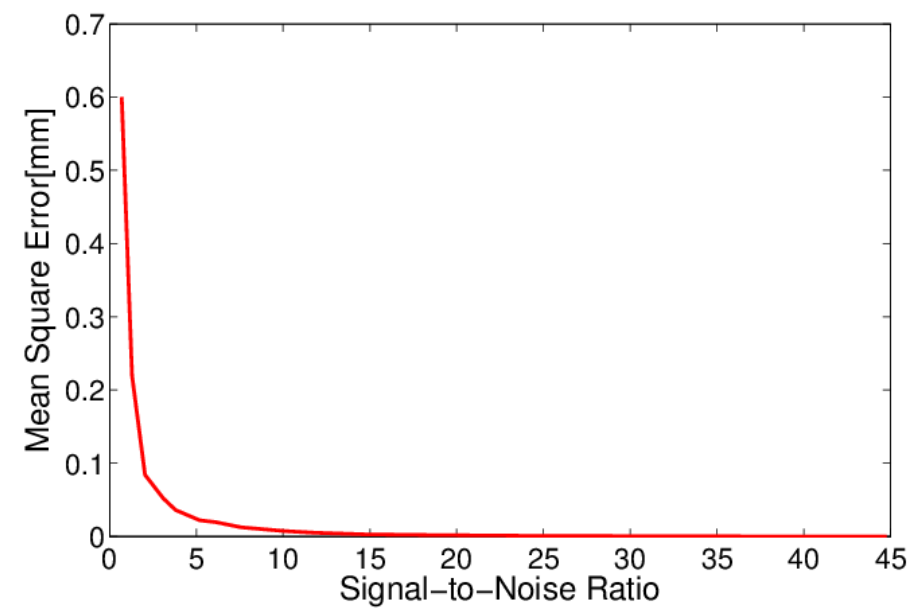

FIG. 3 The relationship between the SNR of the image to register and the MSE of the corresponding estimated vector field with respect to the gold standard.

then pooled such that a joint set containing the displacements from all volunteers at each time point is obtained. Figures 4(a) to 4(f) display this new joint set as an ensemble of boxplots. The displacements have been illustrated separately for the liver and kidneys in the cranio-caudal (CC), left-right (LR) and respectively anterior-posterior (AP) directions. The values on the abscissa correspond to the time delay in minutes since the acquisition of the reference image. The values on the ordinate indicate the estimated signed displacements in millimeters. Each boxplot is constructed in the following fashion: the lower limit of the box corresponds to the first quartile, the upper limit of the box is the third quartile, the median is represented by the red horizontal line in the middle of the box, the red cross indicates the mean of the set, the lower whisker corresponds to the $5^{\text {th }}$ percentile and the upper whisker to the $95^{\text {th }}$ percentile. For both the organs of interest, the overall median of the displacement in the $\mathrm{CC}$ direction has a tendency to increase over time reaching $\sim 3 \mathrm{~mm}$. The maximum liver displacement in the $\mathrm{CC}$ direction is $\sim 8 \mathrm{~mm}$, while for the kidneys this measures $\sim 5 \mathrm{~mm}$. For the LR and AP directions, the median of the displacements does not manifest any tendency. For the liver the maximum displacement in the LR direction is $\sim 3$ $\mathrm{mm}$, while for the kidneys it reaches at a value of $\sim 4 \mathrm{~mm}$. In the AP direction the maximum displacement measures $\sim 2.5 \mathrm{~mm}$ for the liver and $\sim 4 \mathrm{~mm}$ for the kidneys. Figures $4(\mathrm{~g})$ and 4(h) depict the time evolution of the median magnitude of the motion vectors estimated in the liver and respectively kidneys of each volunteer. These graphs reconfirm the tendency of the displacements to increase over time, however, this might happen in non-monotone 
manner, with the organs randomly moving closer or further from their reference position, and with rather large inter-individual variations.

\section{III.D. Validation of the 3D estimated motion}

Validation of the estimated motion was made using the approach described in section II.D.1. Figure 5 displays, for all of the volunteers, the correspondence between the manually determined landmark displacements and the landmark displacements estimated by the optical flow algorithm. The assessment was made in the cranio-caudal (blue circles), left-right (red squares) and anterior-posterior (black crosses) directions respectively. In the case of a perfect correspondence between the manually tracked landmarks and the estimated landmark positions, the points from the graph would lie on the first bisector (depicted by the purple line in figure 5). The precision of the manually tracked displacements is however limited by the discrete nature of the acquired images. Therefore tracking errors of up to one voxel $(2 \mathrm{~mm})$ are to be expected. Nevertheless, a paired T-test applied on the displacements obtained through the two techniques indicates that there is no significant difference between the values at $5 \%$ significance level.

Figure 6 displays positional errors in millimeters that would affect the intervention in the selected landmarks, in the presence and respectively in the absence of a motion correction scheme. The errors were computed as described in section II.D.1. The boxplots are built in the same manner as the ones illustrated in Figure 4. More than $75 \%$ of the errors become less than $2 \mathrm{~mm}$ (one voxel) when motion compensation is present. The median of the errors also drops from $\sim 2.8 \mathrm{~mm}$ to $\sim 1.3 \mathrm{~mm}$.

\section{III.E. In-vivo experiment on a porcine liver}

Figure 7 illustrates the slow physiological drifts experienced by the porcine liver over the duration of the HIFU intervention. The displayed graphs were constructed in a similar fashion as the ones presented in Figure 4. As opposed to the observations made during the volunteer study, there is no clear tendency of the displacements to increase over time in either of the directions. Nevertheless, displacements of up to $4 \mathrm{~mm}$ can be observed in the cranio-caudal direction. 


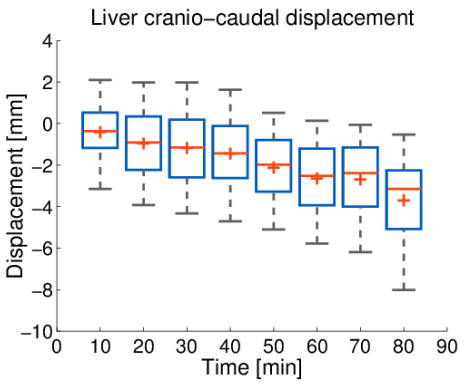

(a)

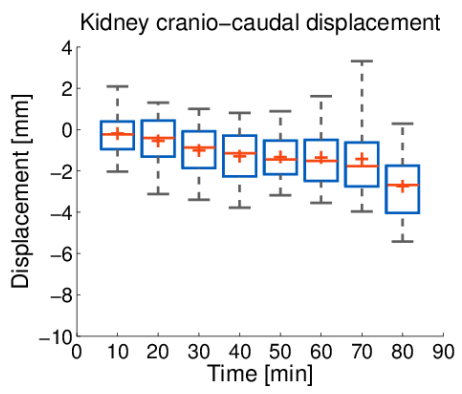

(d)

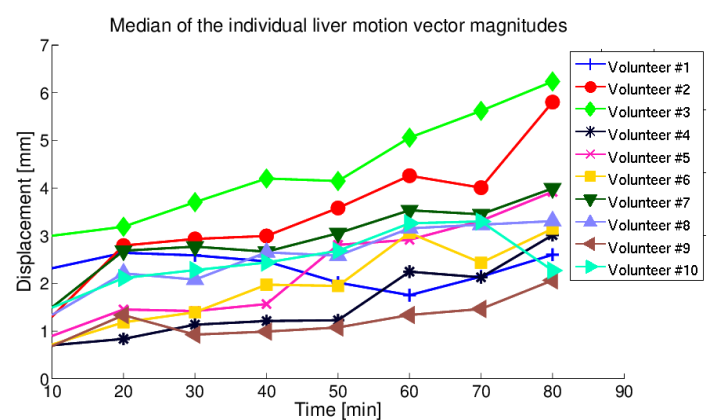

(g)

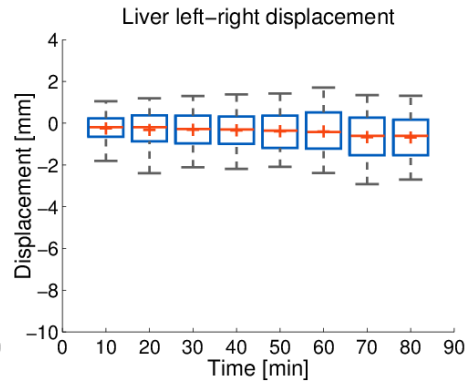

(b)

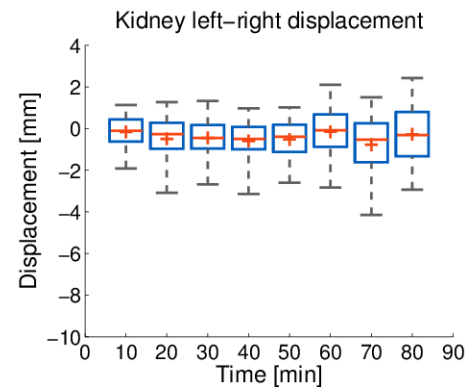

(e)

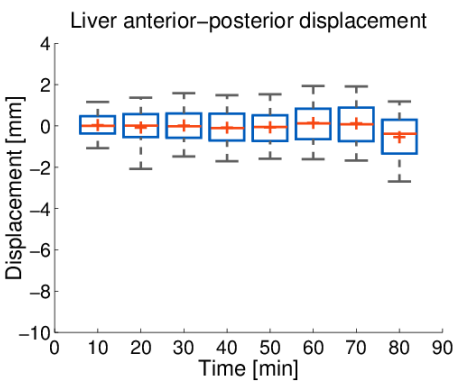

(c)

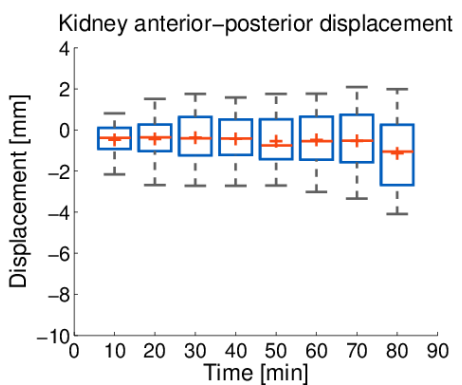

(f)

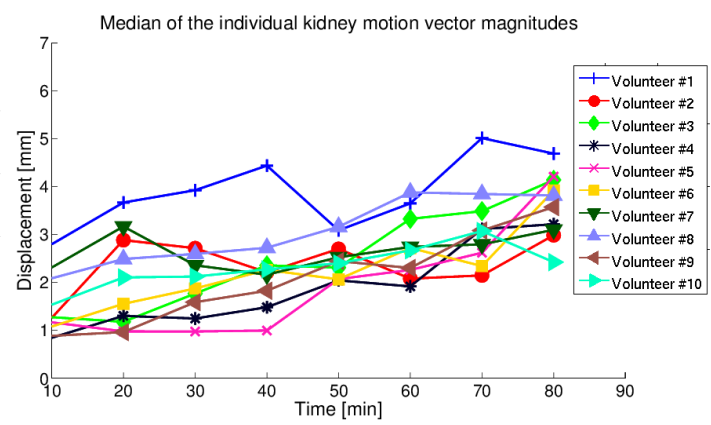

(h)

FIG. 4 Evolution of the liver and kidneys displacement caused by slow physiological motion over a duration of 80 minutes in ten healthy volunteers. (a) - (f) Each boxplot is a representation of a joint set containing the displacements estimated on all volunteers after $k \times 10$ minutes since the acquisition of the $3 \mathrm{D}$ anatomical anchor, with $k=1, \ldots, 8$. (g), (h) The median of the magnitudes of the displacement vectors estimated for the liver and respectively the kidneys of each individual volunteer. The values on the abscissa correspond to the time in minutes since the acquisition of the 3D anatomical anchor. The values on the ordinate are the signed displacements in millimeters in the cranio-caudal, left-right and anterior-posterior directions. 


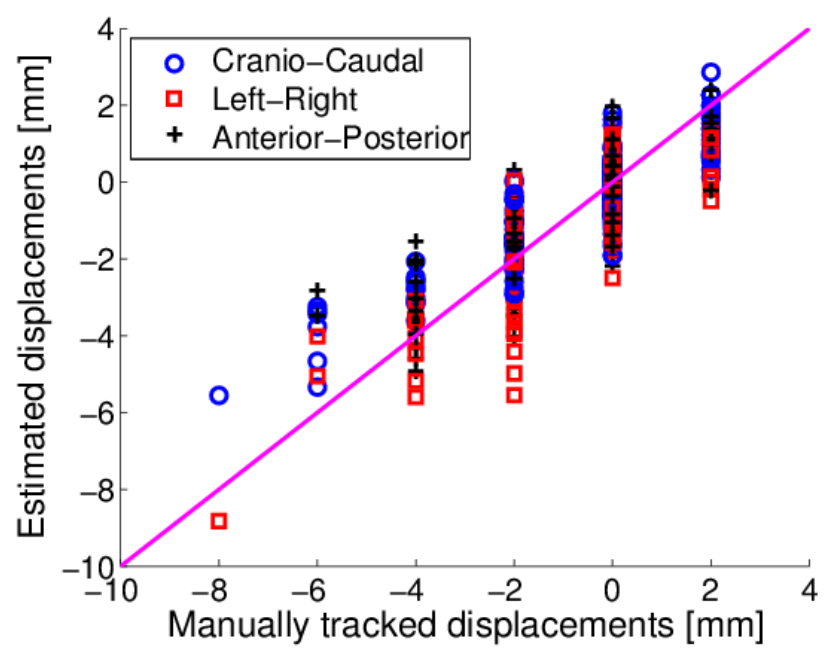

FIG. 5 Validation of the optical flow algorithm. The graph illustrates the correspondence between the displacements obtained by manually tracking a set of vessel bifurcations in the liver and kidneys (on the abscissa) and the displacements estimated by the optical flow algorithm (on the ordinate). The displacements were determined for all volunteers in three directions: cranio-caudal (blue circles), left-right (red squares) and anterior-posterior (black crosses). The purple line corresponds to the ideal location of the plotted points.

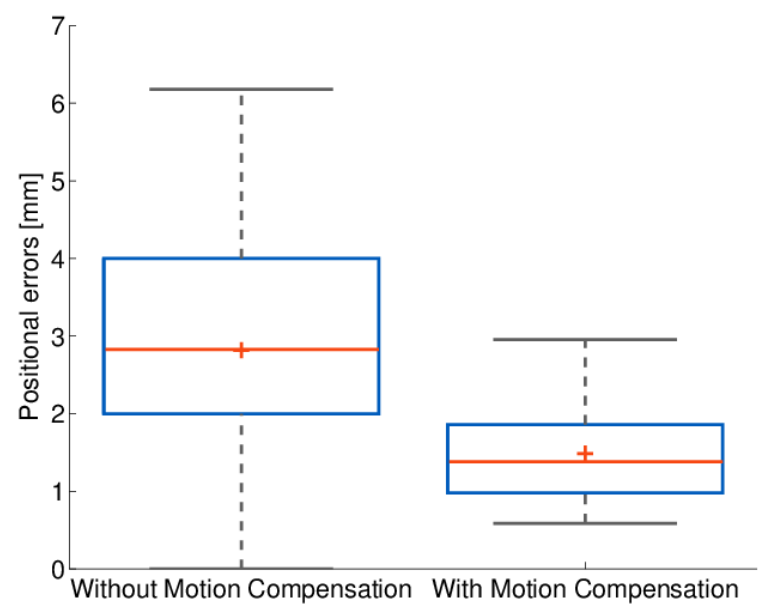

FIG. 6 Positional errors that would affect the intervention in the selected landmarks, in the presence and respectively in the absence of a motion correction scheme. The values on the ordinate correspond to the positional errors in millimeters computed according to II.D.1. 
A magnification of the planning image with focus on the originally planned cell cluster

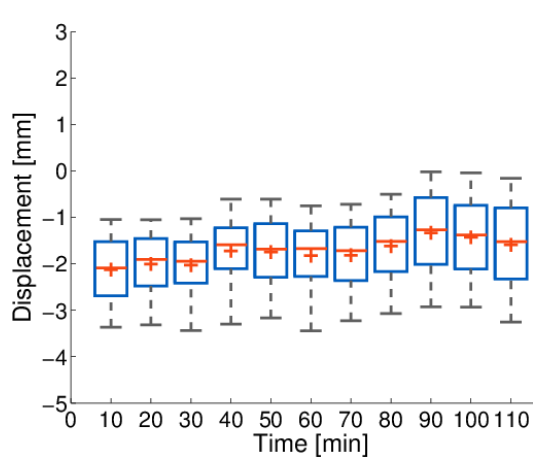

(a)

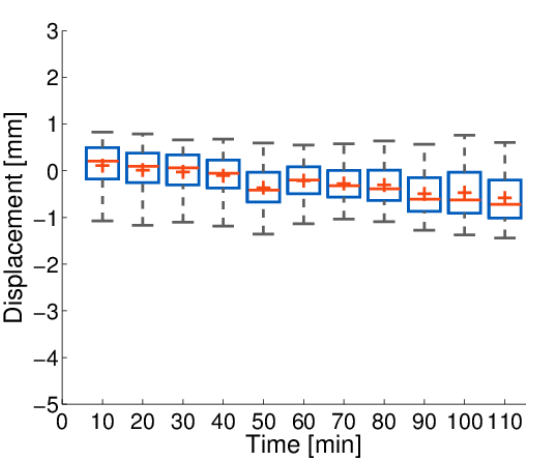

(b)

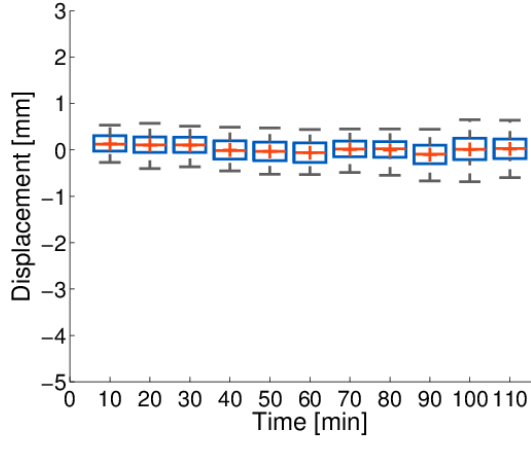

(c)

FIG. 7 Evolution of the liver displacements caused by slow physiological motion during the in-vivo HIFU pig experiment. The values on the abscissa correspond to the time delay in minutes since the acquisition of the 3D anatomical anchor. The values on the ordinate are the signed displacements in millimeters in the (a) cranio-caudal, (b) left-right and (c) anterior-posterior directions.

overlaid as yellow circles is illustrated in figure 8(a). The estimated displacements will allow identifying on the same image the motion corrected sonication cell locations, indicated by the red circles which, due to the organ drifts, will differ from the ones originally planned. The distance between the centers of mass of the planned and the corrected sonication cell clusters measures $\sim 2 \mathrm{~mm}$. The high intensity regions from figures 8 (b) and $8(\mathrm{c})$ depict the anatomy that was determined to have received a lethal thermal dose ${ }^{30}$ without and respectively with motion correction. Without registering the thermometry images with respect to the estimated organ drifts, a positional error will occur between the lethal dose and the corrected location of the sonications. This phenomenon is illustrated in figure 8(b). Figure 8(c) displays the location of lethal thermal dose after registering the thermometry images. The distance between the centers of mass of the position corrected sonication cell cluster and the region determined to have received a lethal thermal dose is reduced from $\sim 2 \mathrm{~mm}$ without motion correction to $\sim 0.3 \mathrm{~mm}$ with motion correction.

Figure 9(a) displays a slice from the contrast enhanced image acquired at the HIFU therapy endpoint. The NPV resulting from the 10 sonications is enclosed in a red rectangle and magnified for better visibility. A mask covering the NPV was manually extracted from this image and overlaid on the planning scan as a high intensity region as depicted in figure 9(b). For positional reference, the corrected sonication locations were also overlaid as red 


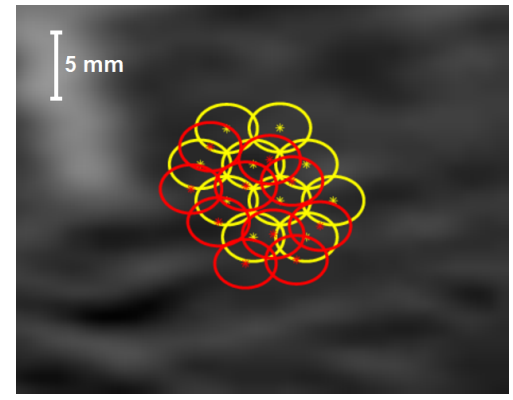

(a)

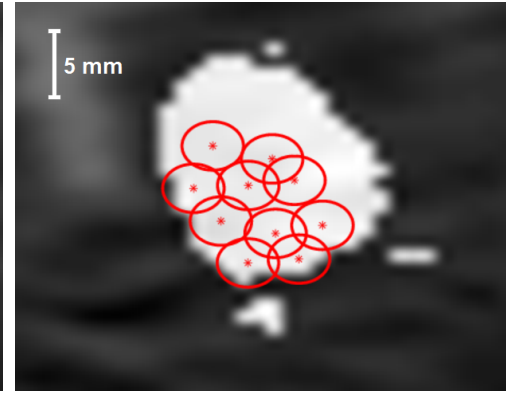

(b)

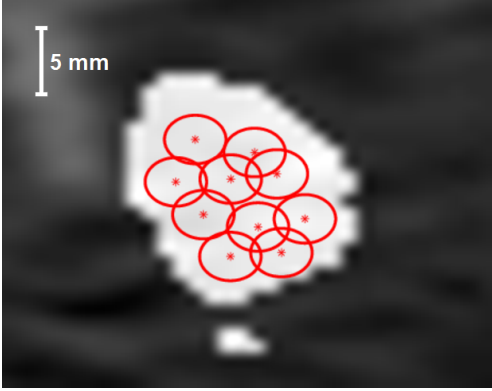

(c)

FIG. 8 (a) Planned cluster of sonication cells (yellow circles) together with the motion corrected location of the sonications (red circles) overlaid on the corresponding anatomy from the planning image. (b), (c) The anatomy that was determined to have received a lethal thermal dose (depicted by the high intensity region): (b) before registering the thermometry images (c) after registering the thermometry images.

circles. A positional discrepancy between the cell cluster and the NPV can be observed. By using the displacements estimated between the last 3D anatomical image and the 3D anatomical anchor, the contrast enhanced image was registered to match the planning image anatomy. The motion corrected NPV was then extracted and superimposed on the planning image as a high intensity region as depicted by figure $9(\mathrm{c})$. Note that due to observer subjectivity during the manual NPV delineation, the non-corrected and the corrected NPV masks might differ. Without motion compensation, the distance between the centers of mass of the position corrected cell cluster and the NPV amounts to $\sim 3.5 \mathrm{~mm}$. By correcting the NPV position the distance is reduced to $\sim 1 \mathrm{~mm}$.

\section{DISCUSSION}

The goal of the presented study was to exploit the episodic work-flow of an MR-guided HIFU therapy to implement a motion correction strategy for the displacement of abdominal organs under the effect of slow physiological drifts. The strategy involves tracking both the target area and organs at risk (OAR) over the entire duration of the intervention.

In the first part of the study we investigated the slowly varying drifts exhibited by the human liver and kidneys during a time period of 80 minutes in 10 healthy volunteers. The obtained displacements of up to $8 \mathrm{~mm}$ for the liver and $5 \mathrm{~mm}$ for the kidneys are in good correspon- 


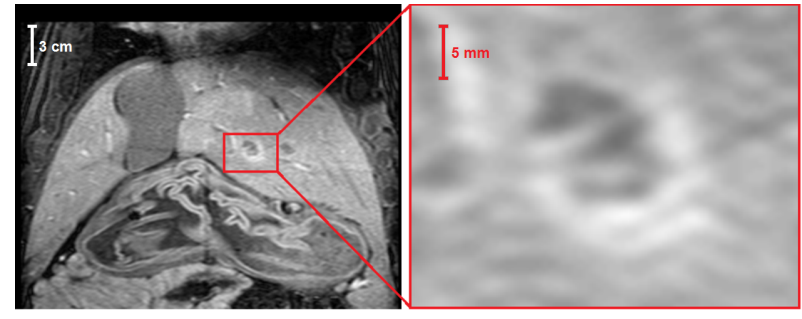

(a)

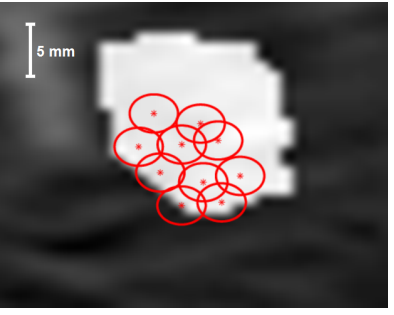

(b)

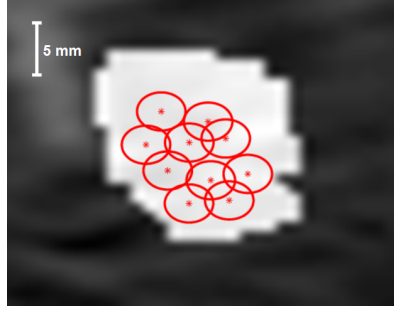

(c)

FIG. 9 (a) Slice of the contrast enhanced scan acquired at the end of the HIFU intervention upon which the resulting NPV is visible. The NPV resulting from the sonications is enclosed in a red rectangle and magnified for better visibility. (b), (c) Corrected sonication locations overlaid as red circles on the corresponding anatomy from the planning image. The high intensity region in the images corresponds to the NPV: (b) without motion correction (c) with motion correction.

dence with previous reportings ${ }^{13,26}$. Please note that respiratory motion was compensated by a navigator gated acquisition scheme for all acquired images. Therefore the obtained shifts are also likely to include shifts of the diaphragm with respect to the navigator.

The findings that result from the volunteer study have shown that large inter-individual variations have to be expected. For the liver of volunteer \#9 the median of the displacements remained close to $1 \mathrm{~mm}$ over the entire 80 minute duration of the study, while for volunteer \#3 the median was already close to $4 \mathrm{~mm}$ halfway through the experiment (see Figure $4(\mathrm{~h})$ ). The measured displacements are also expected to vary with patient position: prone or supine. Thus, this reconfirms that a continuous supervision and correction of the displacements is clearly beneficial for the therapeutic success. For example for the noninvasive treatment of stage 1 tumors in the liver and kidneys the reported shifts represent $\sim 20-50 \%$ of the typical tumor dimensions. If the therapy is conducted without a motion compensation scheme, undesired shifts of this magnitude need to be included in the surgical margin and to a substantially longer intervention ${ }^{31,32}$.

One of the challenges that might occur while evaluating the performance of motion estimation algorithms dedicated to non-invasive in-vivo beam interventions in general is the lack of a reliable gold standard [REF]. For minimally invasive beam therapies this problem was addressed by tracking radio-emitters implanted in/or in the proximity of the targeted region [REF]. Such an approach is, however, not feasible for the applications discussed in this study since it would imply a certain degree of invasiveness. In order to validate the motion esti- 
mated by the employed tracking procedure we have manually tracked a group of landmarks and established the resulting displacements as a gold standard. More than $75 \%$ of the errors between the estimated displacements and the gold standard remained under $2 \mathrm{~mm}$ which is actually the image resolution and thus the precision limit for manual tracking. The source of these errors is the combined effect of misregistration by the motion estimation algorithm and the subjective nature of the landmark manual tracking procedure. It is also expected that as long as the assumptions made by the employed optical flow algorithm are not violated, including voxel gray-level intensity conservation from the reference to the moving image and the continuous nature of the displacements, the same results can be extrapolated to the organs as a whole. Obtaining a gold standard by the proposed approach is however limited by the presence and/or visibility of specific anatomical landmarks (e.g. vessel bifurcations) in the images considered for manual tracking. If this condition is not met, alternative methods need to be considered. Nevertheless, our findings are in good correspondence with previous reportings ${ }^{34-36}$.

The best way to improve the accuracy and precision of the estimated motion is to increase the resolution of the 3D anatomical images used for registration. This however becomes challenging due to the time constraints imposed by the therapy work-flow. If image resolution is increased while keeping the acquisition time at acceptable limits, the SNR of the image might drop to values that deteriorate the estimated motion to the point that the improvement in image resolution becomes practically useless. Accuracy of the estimated motion is also limited by the image distortions intrinsic to the employed MR acquisition sequence. The distortions mainly originate from the inhomogeneities of the MR scanner's static magnetic field and from chemical shifts (ex. water-fat shift) that might occur at organ and body borders. For example, Dragonu et $a l^{37}$ have reported spatial distortions due to $B_{0}$ inhomogeneities larger than $1 \mathrm{~cm}$ in the liver. The study has been performed, however, for rapid gradient recalled MRI sequences using an echo-planar readout, which is not the case in the current work (see section II.C.1). Nevertheless, the results in their study indicate that $B_{0}$ inhomogeneities of $\sim 1.3 \mathrm{ppm}$ are to be expected in the human abdomen. This would cause the sequence used in our study to generate spatial distortions of $\sim 0.3 \mathrm{~mm}$. Such a value is well beneath the image resolution (which is $2 \mathrm{~mm}$ ), thus its effects on the estimated motion were deemed to be negligible. Nevertheless, spatial distortions due to $B_{0}$ inhomogeneities must be addressed for MR images used for motion estimation, either by optimizing 
the MR acquisition sequence or by choosing an appropriate correction scheme ${ }^{38-41}$. In order to eliminate the effects of the water-fat shift, the MR-sequence used in our study has been optimized to be fat suppressing. This will not only remove fat shifts (and thus image artifacts) at the organ and image boundaries but it will also increase the contrast-to-noise ratio of the entire image, which is beneficial for the accuracy of the estimated motion. An additional factor that affects the quality of the estimated motion is the SNR of the images themselves, fact which is to be expected given that the optical flow algorithm employed for registration is gray-level intensity based. According to Figure 3 as the SNR drops bellow 5, the motion estimation errors start increasing exponentially. Thus, it can be considered that beyond this threshold the estimated motion vector field becomes unreliable. This however does not affect in any way the success rate of our method since the 3D anatomical images used for registration have a SNR value of $\sim 44$, therefore in the right extrema of the curve depicted by Figure 3 .

The Horn\&Schunck motion estimation algorithm employed in our study was chosen mainly due to its previous validation for the in-vivo correction of respiratory motion during HIFU ablations in the liver ${ }^{6,25}$. There are, however, a few aspects concerning the algorithm that could be further improved. For example, since it relies on the assumption that voxels conserve their gray-level intensity on the followed trajectory, the Horn\&Schunck algorithm is sensitive to gray-level intensity variations not attributed to motion. Such a situation might occur when, for instance, anatomical structures appear and/or disappear from the field-ofview. In addition, the algorithm imposes that the estimated displacements are spatially continuous. Since abdominal organs are elastic and incompressible, this is a justified assumption. However, an exception from this rule arises at the interface between the organs and the abdominal and/or the thoracic wall. In these regions shearing effects will occur, thus violating the continuity assumption. In order to overcome these limitations, versions of the optical flow algorithm that reduce the impact of such effects could be, for example, employed. Such algorithms have already been applied successfully in the video domain [REF], however, to our knowledge, the validity of such approaches for in-vivo medical imaging applications has yet to be proven.

The focus of this study was on the analysis of the displacements exhibited by the human liver and kidneys due to slow physiological drifts, over the entire duration of a HIFU therapy session. Thus, the MR sequence used to acquire the 3D anatomical images, upon which the 
tracking procedure is based, was optimized to cover the entire upper abdomen. During a real HIFU intervention only the displacements experienced by the tumor itself are of interest. For this purpose the MR sequence can be easily adjusted to the target area and the surrounding organs at risk $(\mathrm{OAR})$ reducing the required acquisition time from $\sim 90 \mathrm{~s}$ to $\sim$ $30 \mathrm{~s}$.

The HIFU experiments performed in-vivo on a porcine liver have shown that the method proposed in our study can be applied for a real HIFU ablation therapy. In the investigated case, the animal was not fed 24 hours prior to the intervention. During the intervention, the animal was under full anesthesia and thus the thoracic and abdominal muscles were under temporary paralysis. Similar to the volunteer experiments, all respiratory induced displacements were compensated by a gating of both all MR-acquisitions and the therapeutic energy delivery. As a consequence, the observed displacements can be largely attributed to shifts due to digestive gas development in the stomach and the intestines. As the timecourse displays (see Figure 7), the majority of the displacement occurred during the first 10 minutes of the intervention. This demonstrates that even under ideal conditions, the occurrence of shifts of 3.5 - $4 \mathrm{~mm}$ can occur and are problematic for therapy. The interventional planning was carried out on a T2-weighted high resolution image obtained at the beginning of the intervention. The subsequent energy deliveries were placed on the target delineation. Therefore, the shift after 10 minutes would have the following consequences for the subsequent therapy :

- Parts of the tumor would have not been ablated, or the surgical margin would have not been sufficient.

- Shifts in-between shots potentially lead to small areas of viable tissue in between the ablation cells. Without motion compensation these have to be accounted for by a large overlap of the sonication cells, which significantly decreases the achievable ablation speed and thus the overall duration of the intervention.

It is important to note that during the in-vivo experiment no motion estimation or correction has been performed. The estimation of the displacements was made in post-processing using the 3D anatomical images acquired during therapy. The resulting motion vectors were used to retrospectively update the position and configuration of the sonication cell cluster such that it will match the actual ablated anatomy. The small residual shift of $\sim 0.3 \mathrm{~mm}$ 
between the updated cell cluster and the motion corrected thermal dose and of $\sim 1 \mathrm{~mm}$ between the updated cell cluster and the motion corrected NPV is a good indicator that the proposed motion estimation procedure can be successfully applied in-vivo with acceptable error margins.

The current multithreaded $\mathrm{C}++$ implementation of the $3 \mathrm{D}$ registration algorithm used in our study has an execution time of approximately $30 \mathrm{~s}$. Although motion compensation was performed in post-processing, the time interval between energy deliveries, which is usually 6-8 minutes, allows an integration. Furthermore, acceleration using an implementation via the compute unified device architecture (CUDA) platform on a graphical processing unit $(\mathrm{GPU})^{25}$ allows to bring the motion estimation down to $\sim 10$ seconds, which renders the approach feasible with the overall work-flow.

The motion compensation framework proposed in this study is, however, not limited to HIFU interventions. With the development of the first MR linear accelerator (MR-LinAc) prototypes[REF], our motion correction strategy could potentially be integrated in the work-flow of an MR-guided radiotherapy (MRg-RT). Similar to HIFU interventions, energy delivery during external beam radiotherapy is usually episodic, with the total prescribed radiation dose being divided into multiple fractions. The time interval in between fractions, which can sometimes extend over several days, allows the healthy tissues to partially recover from the effects of the absorbed radiation. These time intervals can be seen as the equivalent of the cool-down periods during HIFU therapies. Naturally, there is a high probability that due to the discussed physiological drifts or due to patient positional errors, the target location will differ from one fraction to the next. If these displacements are not taken into consideration, they might lead to the under-treatment of the tumor or to additional damage to the OAR's. To address this problem, a 3D anatomical image could be acquired, for example, after each fraction and registered to a 3D anatomical anchor acquired before delivering the first fraction. The resulting motion vector fields could then be integrated into the therapy planning algorithm and the plan could be re-optimized prior to the next fraction. The proposed framework can also be adapted to operate during the delivery of a single fraction. In order to reduce the damage caused to the OAR's, a single fraction is usually delivered over several changes in the gantry position. A 3D anatomical image could be acquired while changing gantry positions and registered to a 3D anatomical anchor acquired prior to starting dose delivery. The estimated displacements could be used, for example, to re-optimize the plan 
on-the-fly. However, such an approach is timewise challenging since the gantry positional changes usually take place over time intervals of only several seconds.

\section{ACKNOWLEDGMENTS}

This work was supported in part by the European Research Council (project ERC-2010555

So far, studies concerned with motion estimation during MRg-HIFU interventions in the abdomen were focused on tracking the targeted area only over the time course of the therapeutic energy delivery. At this level, the interest was compensating for motion mainly caused by respiration. In the presented study, we demonstrated that motion compensation only during energy delivery is conceptually not sufficient : HIFU therapies rely on a non-invasive target identification and delineation, the subsequent interventional planning of the ablation areas, the therapeutic energy delivery and the validation of the therapeutic endpoint. One of the strengths of MR-guided interventions is that except the initial target identification and delineation, all subsequent steps can be carried out on-the-fly with the patient in place on the interventional table. The advantage of the proposed approach is that MR-guided interventions allow to adjust planning and therapy directly to the current anatomical position for the particular position of the patient on the interventional table. While static parts of the human anatomy such as the cranium or the limbs are relatively easy to immobilize (stereotactic frames, limb molds), the thoracic and the abdominal area are subject to both respiratory motion and slow physiological drifts. As we have demonstrated, this can lead to a misalignment between interventional planning, energy delivery and therapeutic validation. Since this can potentially lead to both undesired tissue damage of organs at risk and viable residual tumor tissue, a suitable motion correction strategy should encompass all of the essential steps of the intervention: Planning, monitoring of the therapeutic energy delivery and the validation of the therapeutic endpoint. The presented method is a first step towards such a motion correction strategy for interventions in the abdomen.

\footnotetext{
AdG-20100317, Sound Pharma) and in part by the Dutch Technology Foundation (STW)
}

\section{CONCLUSIONS}


(project OnTrack \#12813).

\section{REFERENCES}

${ }^{1}$ H. Cline, J. Schenck, K. Hynynen, R. Watkins, S. Souza, and F. Jolesz, "MR-guided facused ultrasound surgery," J Comput Assist Tomogr 16, 956-965 (1992).

${ }^{2}$ K. Hynynen, W. Freund, H. Cline, A. Chung, R. Watkins, J. Vetro, and F. Jolesz, "A clinical, noninvasive, MR imaging-monitored ultrasound surgery method," Radiographics 16, 185-195 (1996).

${ }^{3}$ R. Illing, J. Kennedy, G. ter Haar, A. Protheroe, P. Friend, F. Gleeson, D. Cranston, R. Phillips, and M. Middleton, "The safety and feasibility of extracorporeal high-intensity focused ultrasound (hifu) for the treatment of liver and kidney tumours in a western population," Br J Cancer 93, 890-895 (2005).

${ }^{4}$ C. Tempany, N. McDannold, K. Hynyen, and F. Jolesz, "Focused ultrasound surgery in oncology: overview and principles," Radiology 36, 39-56 (2011).

${ }^{5}$ J. Foley, M. Eames, J. Snell, A. Hananel, N. Kassell, and J.-F. Aubry, "Image-guided focused ultrasound: state of the technology and the challanges that lie ahead," Imaging Med. 5, 357-370 (2013).

${ }^{6}$ M. Ries, B. Denis de Senneville, S. Roujol, Y. Berber, B. Quesson, and C. Moonen, "Realtime 3D target tracking in MRI guided focused ultrasound ablations in moving tissues," Magnetic Resonance in Medicine 64, 1704-1712 (2010).

${ }^{7}$ S. Crijns, B. Raaymakers, and J. Lagendijk, "Proof of concept of MRI-guided tracked radiation delivery: tracking one-dimensional motion," Physics in Medicine and Biology 57, 7863-7872 (2012).

${ }^{8}$ S. Diez, J. Garcia, and F. Sendra, "Analysis and evaluation of periodic physiological organ motion in radiotherapy treatments," Radiotherapy and Oncology 73, 325-329 (2004).

${ }^{9}$ M. Goitein, "Organ and tumor motion: An overview," Seminars in Radiation Oncology $14,2-9(2004)$.

${ }^{10}$ S. Vijayan, S. Klein, E. Hofstad, F. Lindseth, B. Ystgaard, and T. Lango, "Validation of a non-rigid registration method for motion compensation in 4D ultrasound of the liver," IEEE 10th International Symposium on Biomedical Imaging , 792-795 (2013). 
${ }^{11} \mathrm{~J}$. Booth, Modelling the impact of treatment uncertainties in radiotherapy, Ph.D. thesis, University of Adelaide (2002).

${ }^{12}$ K. Langen and D. Jones, "Organ motion and its management," Int J Radiation Oncology Biol Phys 50, 265-278 (2001).

${ }^{13}$ M. von Siebenthal and G. Szekely, "Systematic errors in respiratory gating due to intrafraction deformations in the liver," Med. Phys. 34, 265-278 (2001).

${ }^{14}$ K. Langen, T. Willoughby, S. Meeks, A. Santhanam, A. Cunningham, L. Levine, and P. Kupelian, "Observations on real-time prostate gland motion using electromagnetic tracking," Int J Radiation Oncology Biol. Phys. 71, 1084-1090 (2008).

${ }^{15}$ P. Arnold, F. Preiswerk, B. Fasel, R. Salomir, K. Scheffler, and P. Cattin, "3D organ motion prediction for MR-guided high intensity focused ultrasound," Med Image Comput Comput Assist Interv 14, 623-630 (2011).

${ }^{16}$ M. Smitmans, F. Pos, J. de Bois, W. Heemsbergen, J.-J. Sonke, J. Lebesque, and M. van Herk, "The influence of a dietary protocol on a cone beam CT-guided radiotherapy for prostate cancer patients," Int J Radiation Oncology Biol Phys 71, 1279-1286 (2008).

${ }^{17}$ J. Emmott, B. Sanghera, J. Chambers, and W. Wong, "The effects of n-butylscopolamine on bowel uptake: an F-FDG PET study," Nuclear Medicine Communications 29, 11-16 (2008).

${ }^{18}$ A. Padhani, V. Khoo, J. Suckling, P. Minst, J. Husband, M. Leach, and D. Dearnaley, "Evaluating the effect of rectal distension and rectal movement on prostate gland position using cine MRI," Int J Radiation Oncology Biol Phys 44, 525-533 (1999).

${ }^{19}$ R. Mirabell, P. Nouet, M. Rouzaud, A. Bardina, N. Heijira, and D. Schneider, "Radiotherapy of bladder cancer: relevance of bladder volume changes in planning boost treatment," Int J Radiation Oncology Biol Phys 41, 741-746 (1998).

${ }^{20}$ J. Thariat, S. Aluwini, and M. Housset, "Adaptive partial-boost stereotactic radiation therapy for muscle-invasive carcinoma of the urinary bladder," in Robotic Radiosurgery. Treating prostate cancer and related genitourinary applications, edited by L. E. Ponsky, D. Fuller, R. Meier, and C. Ma (Springer Berlin Heidelberg, 2012) pp. 185-200.

${ }^{21}$ L. Verhey, "Immobilizing and positioning patients for radiotherapy," Seminars in radiation oncology 5, 100-114 (1995).

${ }^{22}$ Y.-F. Zhou, "High intensity focused ultrasound in clinical tumor ablation," World J Clin Oncol 2, 8-27 (2011). 
${ }^{23}$ L. Zhang, W.-Z. Chen, Y.-J. Liu, X. Hu, K. Zhou, S. Chen, L. Peng, H. Zhu, H.-L. Zou, J. Bai, and Z.-B. Wang, "Feasibility of magnetic resonance imaging-guided high intensity focused ultrasound therapy for ablating uterine fibroids in patients with bowel lies anterior to uterus," Eur J Radiol 73, 396-403 (2010).

${ }^{24}$ B. Denis de Senneville, M. Ries, G. Maclair, and C. Moonen, "Mr-guided thermotherapy of abdominal organs using a robust PCA-based motion descriptor," IEEE Trans Med Imaging 30, 1987-1995 (2011).

${ }^{25}$ S. Roujol, M. Ries, B. Quesson, C. Moonen, and B. Denis de Senneville, "Real-time MRthermometry and dosimetry for interventional guidance on abdominal organs," Magnetic Resonance in Medicine 63, 1080-1087 (2010).

${ }^{26}$ R. Case, J.-J. Sonke, D. Moseley, J. Kim, K. Brock, and L. Dawson, "Inter- and intrafraction variability in liver position in non-breath-hold stereotactic body radiotherapy," Int J Radiation Oncology Biol Phys 75, 302-308 (2009).

${ }^{27}$ C. Damianou and K. Hynynen, "Focal spacing and near-field heating during pulsed hightemperature ultrasound therapy," Ultrasound Med Biol 19, 777-787 (1993).

${ }^{28}$ B. Horn and B. Schunck, "Determining optical flow," Artificial Intelligence 17, 185-203 (1981).

${ }^{29}$ T. Brox, A. Bruhn, N. Papenberg, and J. Weickert, "High accuracy optical flow estimation based on a theory for warping," in Proc. 8th European Conference on Computer Vision, Vol. 4 (Springer, 2004) pp. 25-34.

${ }^{30} \mathrm{~S}$. Sapareto and W. Dewey, "Thermal dose determination in cancer therapy," Int. J. Radiation Oncology Biol. Phys. 10, 787-800 (1984).

${ }^{31} \mathrm{~F} . \mathrm{Wu}$, "Extracorporeal high intensity focused ultrasound in the treatment of patients with solid malignancy," Minimally Invasive Therapy 15, 26-35 (2006).

${ }^{32}$ G. S.N., G. Gazelle, and P. Mueller, "Thermal ablation therapy for focal malignancy: A unified approach to underlying principles, techniques, and diagnostic imaging guidance," American Journal of Roentgenology 174, 323-331 (2000).

${ }^{33}$ G. Zhang, H.-H. M. Yu, C. Stevens, T. Diling, S. Hoffe, E. Moros, and V. Feygelman, "Motion management in stereotactic body radiotherapy," J Nucl Med Radiat Ther S6, $012(2012)$.

${ }^{34}$ N. Kadoya, Y. Fujita, Y. Katsuta, S. Dobashi, K. Takeda, K. Kishi, M. Kubozono, R. Umezawa, T. Sugawara, H. Matsushita, and K. Jingu, "Evaluation of various de- 
formable image registration algorithms for thoracic images," Journal of Radiation Research $650 \quad, 1-8(2013)$.

${ }^{35}$ R. Castillo, E. Castillo, R. Guerra, V. E. Johnson, T. McPhail, A. Garg, and T. Guerrero, "A framework for evaluation of deformable image registration spatial accuracy using large landmark point sets," Phys. Med. Biol 54, 1849-1870 (2009).

${ }^{36} \mathrm{~K}$. Brock, "Results of a multi-institution deformable registration accuracy study(MIDRAS)," Int J Radiation Oncology Biol. Phys. 76, 583-596 (2010).

${ }^{37}$ J. Dragonu, B. Denis de Senneville, B. Quesson, C. Moonen, and M. Ries, "Real-time geometric distorsion correction for interventional imaging with echo-planar imaging (EPI)," Magnetic Resonance in Medicine 61, 994-1000 (2009).

${ }^{38} \mathrm{H}$. Chang and J. M. Fitzpatrick, "A technique for accurate magnetic resonance imaging in the presence of field inhomogeneities," IEEE Trans Med Imaging 11, 319-329 (1993).

${ }^{39}$ T. Sumanaweera, G. Glover, T. Binford, and J. Adler, "MR susceptibility misregistration correction," IEEE Trans Med Imaging 12, 251-259 (1993).

${ }^{40}$ T. Sumanaweera, J. Adler, S. Napel, and G. Glover, "Characterization of spatial distorsion in magnetic resonance imaging and its implications for stereotactic surgery," Neurosurgery 35, 696-703 (1994).

${ }^{41}$ S. Reinsberg, S. Doran, E. Charles-Edwards, and M. Leach, "A complete distorsion correction for MR images: II. Rectification of static field inhomogeneities by similarity-based profile mapping," Med Phys Biol 50, 2651-2661 (2005). 